\title{
TEM analysis of dislocation structures formed in the Cr-doped grain boundary of alumina
}

\author{
Yuki KEZUKA, ${ }^{*}$ Eita TOCHIGI, ${ }^{*}$ Naoya SHIBATA ${ }^{*}$ and Yuichi IKUHARA ${ }^{* * *, * *, \dagger}$
}

${ }^{*}$ Institute of Engineering Innovation, The University of Tokyo, Bunkyo, Tokyo 113-8656

${ }^{* *}$ Nanostructures Research Laboratory, Japan Fine Ceramics Center, Nagoya 456-8587

*** WPI-AIMR, Tohoku University, Sendai 980-8577

\begin{abstract}
An alumina bicrystal with a $\mathrm{Cr}$-doped $\{1 \overline{100}\} /[0001] 2^{\circ}$ low-angle tilt grain boundary was fabricated by diffusion bonding at elevated temperatures, and the microstructures around the grain boundary were observed by transmission electron microscopy. It was confirmed that an approximately $200 \mathrm{~nm}$ width $\mathrm{Cr}$-rich secondary phase was formed along the grain boundary. Not only an array of triply dissociated dislocations was introduced along the grain boundary but also misfit dislocations were introduced along the heterointerface between the $\mathrm{Cr}$-rich secondary phase and the alumina matrix. By observing the heterointerface from the $\langle 1100\rangle$ direction which is perpendicular to the plane, it was found that all the misfit dislocations dissociate into partial dislocations. The dissociation width was several times larger than that in alumina $\{1 \overline{100}\}$ planes reported so far.
\end{abstract}

(2011 The Ceramic Society of Japan. All rights reserved.

Key-words: Alumina, Ruby, Grain boundary, Dislocation, Stacking fault, Heterointerface, Bicrystal, Transmission electron microscopy (TEM)

[Received July 20, 2011; Accepted August 3, 2011]

\section{Introduction}

Sapphire doped with $\mathrm{Cr}^{3+}$ is well known as ruby, which exhibits two sharp luminescent peaks of R1 $(694.3 \mathrm{~nm})$ and R2 $(692.9 \mathrm{~nm})$ in the red portion of the visible light range. ${ }^{1), 2}$ Since the late 1950 s, many studies have been conducted to investigate its optical properties because of its importance as the firstly discovered laser material. ${ }^{3)-5)}$ The wavelengths of these two sharp luminescent peaks are sensitive to stress ${ }^{(6)-12)}$ and temperature, ${ }^{13), 14)}$ and thus ruby has been applied for stress sensors and fluorescence thermometers which can be used even under elevated temperatures.

In recent years, the fabrication of nanostructured alumina such as thin films, ${ }^{15)-17)}$ nanowires, ${ }^{18)}$ and nanobelts ${ }^{19), 20)}$ have been studied extensively. When these nanostructures contain $\mathrm{Cr}^{3+}$, they exhibit luminescent properties. ${ }^{15), 18)}$ For instance, N. Yu et al. reported the fabrication of ruby thin films on surfaces of $\alpha-\mathrm{Al}_{2} \mathrm{O}_{3}$ substrates and their fluorescence properties. ${ }^{15)}$ On the other hand, in our earlier work, we have established a successful method to introduce thin ruby phases along grain boundaries of alumina by joining metallic Cr-coated alumina single crystals at high temperatures, and investigated the luminescent properties of these phases using confocal micro-luminescence spectroscopy. ${ }^{21)}$ By introducing these thin phases inside alumina, it will be more resistant to scratch, stain or decay, and the application fields will further be expanded. It was also confirmed that not only grain boundary dislocations were introduced to compensate for the mistilt angle of the boundary but also misfit dislocations were introduced at the interface between the Cr-rich secondary phase and the alumina matrix.

In the present study, these dislocation structures were investigated in detail from a cross-sectional direction and a plan-view direction using transmission electron microscopy (TEM).

Corresponding author: Y. Ikuhara; E-mail: ikuhara@sigma.t. u-tokyo.ac.jp

\section{Experimental procedure}

The $\{1 \overline{1} 00\} /[0001] 2^{\circ}$ low-angle tilt grain boundary was chosen as a model grain boundary of alumina, and a Cr-doped bicrystal was fabricated as follows. Two blocks of high-purity alumina single crystals ( $>99.99 \%$, Shinkosha Co. Ltd.) with the size of $15 \mathrm{~mm} \times 12 \mathrm{~mm} \times 6 \mathrm{~mm}$ were used as the starting materials. The surfaces of the two crystals were polished by the mechanochemical polishing using colloidal silica, ${ }^{22)}$ and then metallic $\mathrm{Cr}$ was deposited onto a surface of one crystal by vacuum evaporation. The two crystals were subsequently joined at $1500^{\circ} \mathrm{C}$ for $10 \mathrm{~h}$ in air with the approximate cooling rate of $5^{\circ} \mathrm{C} / \mathrm{min}$. The schematic illustration of the bicrystal fabricated in this study is shown in Fig. 1(a). From thus fabricated bicrystal, two different types of plates were mechanically prepared: one is for the cross-sectional observation and the other is for the planview observation. Both types of plates had the approximate size of $2 \mathrm{~mm} \times 2 \mathrm{~mm} \times 20 \mu \mathrm{m}$. The schematic illustrations of the prepared plates are shown in Figs. 1(b) and 1(c). After polishing both surfaces of these plates, ion-beam milling with $\mathrm{Ar}^{+}$ions from both sides was carried out to obtain electron transparency. Inclination angles of incident $\mathrm{Ar}^{+}$ions were set $8^{\circ}$, and the ion energy was set $4 \mathrm{kV}$.

The grain boundary structures of the prepared thin samples were observed by conventional TEM (JEOL JEM-2010HC, operated at $200 \mathrm{kV}$ ) and high resolution TEM (JEOL JEM-4010, operated at $400 \mathrm{kV}$ ). The composition around the grain boundary was analyzed quantitatively using TEM-Energy dispersive X-ray spectroscopy (EDS) (Topcon EM-002BF, operated at $200 \mathrm{kV}$ ) with the probe size of approximately $1.5 \mathrm{~nm}$.

\section{Results and discussion}

Figure 2(a) is a low-magnification bright-field TEM image of the grain boundary. The observation was performed form the [0001] zone axis and the corresponding selected-area electron diffraction pattern is shown in Fig. 2(b). It can be seen that 

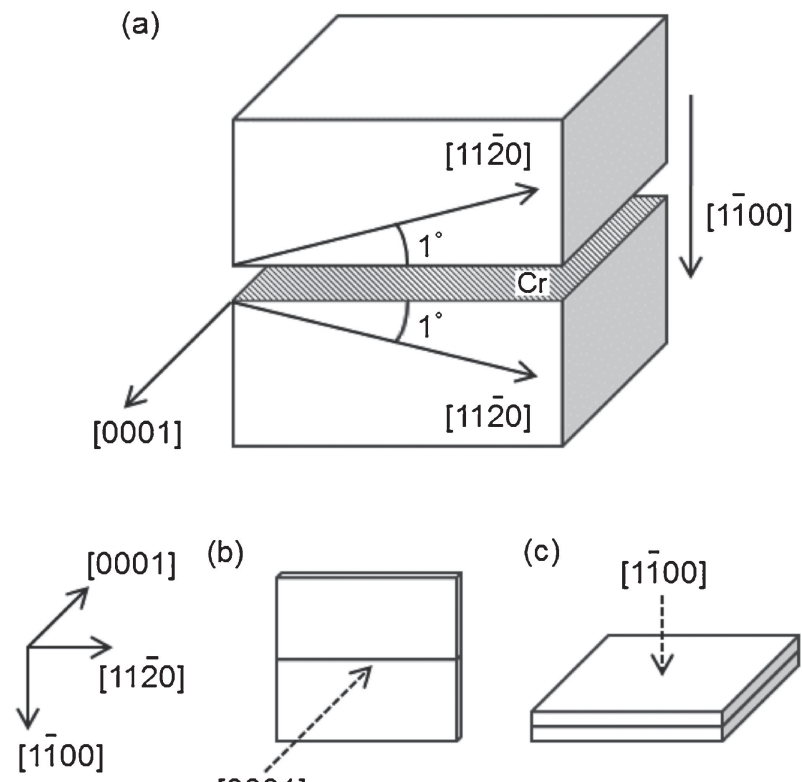

(b)

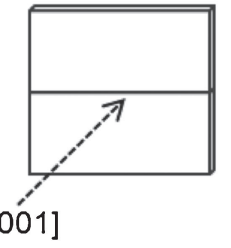

(c)

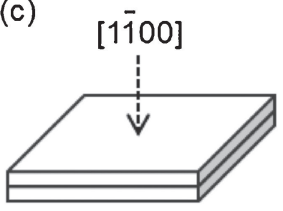

Fig. 1. Schematic illustrations of (a) the alumina bicrystal with Cr-doped $\{1 \overline{1} 00\} /[0001] 2^{\circ}$ low-angle tilt grain boundary, (b) TEM sample for cross-sectional observation and (c) TEM sample for plan-view observation.

Cr-rich secondary phase of about $200 \mathrm{~nm}$ width is formed along the grain boundary. Here, the diffusion distance of $\mathrm{Cr}$ atoms in alumina under the bonding condition in this study (i.e., at $1500^{\circ} \mathrm{C}$ for $10 \mathrm{~h}$ in air), $(D t)^{1 / 2}$, is estimated to be $\sim 0.12 \mu \mathrm{m}$ using the reported volume diffusion coefficient, $D$, of $\sim 4.3 \times 10^{-19} \mathrm{~m}^{2} \mathrm{~s}^{-1}$ (at $\left.1500^{\circ} \mathrm{C}\right){ }^{23), 24)}$ Therefore, the width of the Cr-rich secondary phase is reasonable under the present experimental bonding condition. From the electron diffraction pattern, the tilt angle, $2 \theta$, of the grain boundary is measured to be $1.9^{\circ}$ as indicated by inset in Fig. 2(b). It is also confirmed that the Cr-rich secondary phase has the corundum structure, the same structure with the alumina matrix. In the microgragh, the dot-like strain contrasts are formed between the Cr-rich secondary phase and the alumina matrix as indicated by the solid arrow, and these contrasts must be originated from the misfit dislocations introduced to compensate for the lattice mismatches between Cr-rich secondary phase and the alumina matrix.

It is also noticed from the Fig. 2(a) that a wavy array of dislocations is formed at the center of the Cr-rich secondary phase as indicated by the dotted arrow. This is the array of grain boundary dislocations introduced due to compensate for the mistilt angle of the boundary. Figure 3(a) is a magnified bright field TEM image of the dislocation array. It can be seen that the dislocation triplets are periodically introduced along the grain boundary with the interval, $d$, of about $25 \mathrm{~nm}$. Since the tilt angle $2 \theta$ of the grain boundary is measured to be $1.9^{\circ}$, the magnitude of the Burgers vector, $b$, of the perfect dislocations is estimated approximately to be $0.83 \mathrm{~nm}$ by the geometrical relationship known as Frank's formula: ${ }^{25)}$

$$
\mathrm{b}=2 \theta \times d
$$

The smallest translation vector perpendicular to the $\{1 \overline{1} 00\}$ plane is $\langle 1 \overline{1} 00\rangle$, and the magnitude of which is $0.824 \mathrm{~nm}$. Therefore, the Burgers vector of the perfect dislocations introduced along the grain boundary should be $\langle 1 \overline{1} 00\rangle$. Figure $3(\mathrm{~b})$ is a high resolution TEM image of a dislocation triplet. Burgers circuits around each (a)

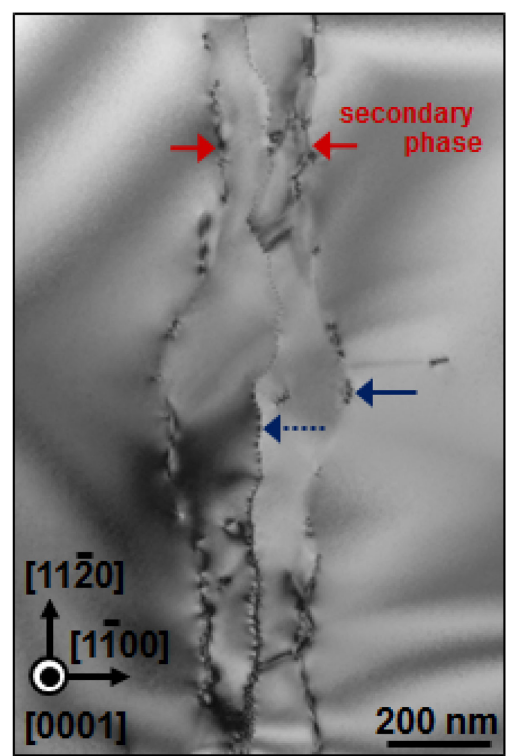

(b)

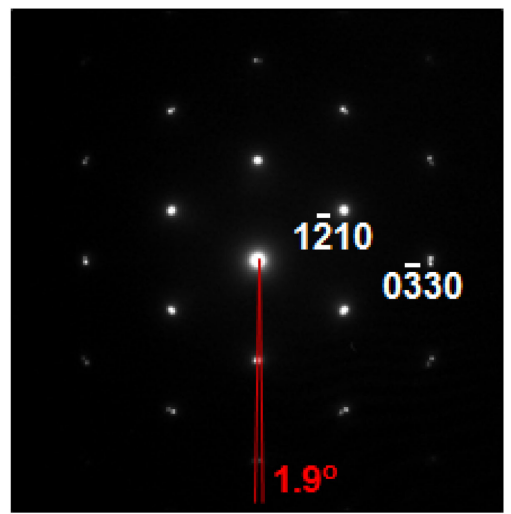

Fig. 2. (Color online) (a) Low-magnification bright-field TEM image of the grain boundary observed from the cross-sectional direction of [0001]. The formation of a secondary phase along the grain boundary is confirmed. (b) Selected-area electron diffraction pattern obtained from the region including the secondary phase.

partial dislocations are shown in this image, and the Burgers vector of each partial dislocation is identified to be $1 / 3\langle 1 \overline{1} 00\rangle$. This result indicates that the perfect dislocations introduced in $\{1 \overline{1} 00\} /[0001]$ low-angle tilt grain boundary dissociate into three partial dislocations according to the following equation:

$$
\langle 1 \overline{1} 00\rangle \rightarrow 1 / 3\langle 1 \overline{1} 00\rangle+1 / 3\langle 1 \overline{1} 00\rangle+1 / 3\langle 1 \overline{1} 00\rangle
$$

Although the Burgers vector $\langle 1 \overline{1} 00\rangle$ of the perfect dislocations coincides with perfect translation vectors both in the anion sublattice and in the cation sublattice, the Burgers vector $1 / 3\langle 1 \overline{1} 00\rangle$ of the dissociated partial dislocations does not coincide the perfect translation vectors in the cation sublattice. Therefore, stacking faults should be introduced along the grain boundary on the $\{1 \overline{1} 00\}$ plane, in between the dissociated partial dislocations.

To investigate the atomic composition around the Cr-rich secondary phase region, TEM-EDS point analysis was conducted. Figure 4(a) shows typical spectrum obtained from an area inside the Cr-rich secondary phase region and Fig. 4(b) shows spectrum obtained from an area approximately $3 \mathrm{~nm}$ outside of the Cr-rich secondary phase-alumina heterointerface. Cr-induced peaks of $\mathrm{CrK}_{\alpha}$-line and $\mathrm{CrK}_{\beta}$-line are clearly observed in the spectrum (a), whereas not in the spectrum (b). 

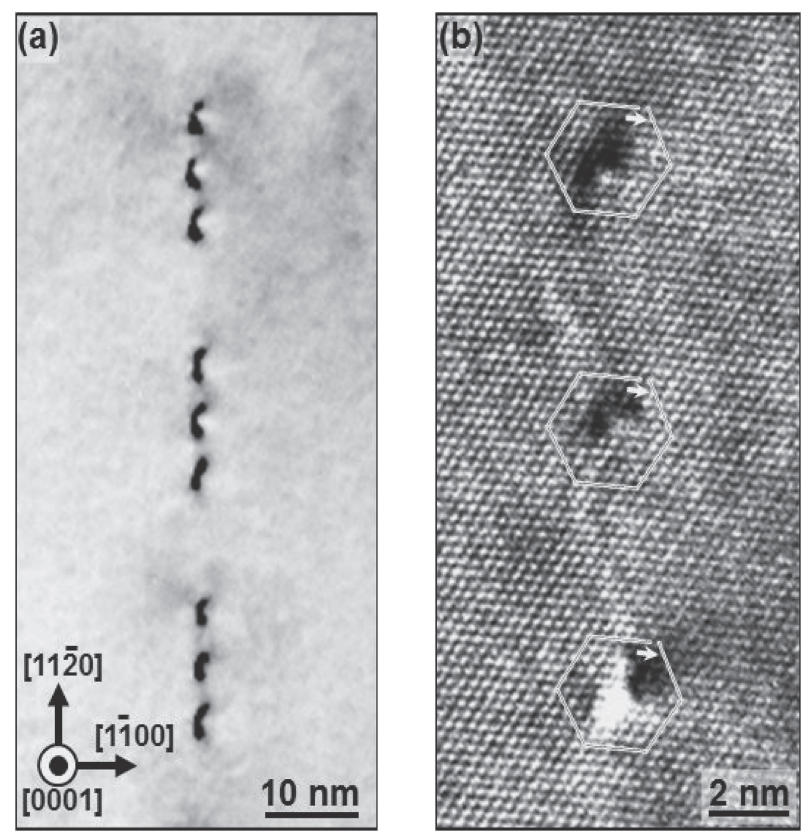

Fig. 3. (a) Magnified bright field TEM image of the dislocation array (cross-sectional observation). Introduction of dislocation triplets with the interval of approximately $25 \mathrm{~nm}$ can be observed. (b) High resolution TEM image of the dislocation triplet. The Burgers circuits indicate that each dislocation has the edge component of $1 / 3\langle 1 \overline{1} 00\rangle$.

$\mathrm{Cr}$ concentration in the Cr-rich secondary phase was estimated as follows. Ten typical spectra obtained from the Cr-rich secondary phase region was chosen, $\mathrm{CrK}_{\alpha}$-lines and $\mathrm{AlK}_{\alpha}$-lines were used for the quantitative analysis and the average peak intensity ratio $I_{\mathrm{Cr}} / I_{\mathrm{Al}}$ was calculated to be 0.128 . Supposing that the sample thickness is very thin, the weight concentration ratio of cations $C_{\mathrm{Cr}} / C_{\mathrm{Al}}$ can be calculated to be 0.118 with the following equation: ${ }^{26)}$

$$
C_{\mathrm{Cr}} / C_{\mathrm{Al}}=k_{\mathrm{CrAl}}\left(I_{\mathrm{Cr}} / I_{\mathrm{Al}}\right)
$$

where the Cliff-Lorimer factor, $k_{\mathrm{CrAl}}$, is 0.917 in this case. Thus, it can be estimated that the $\mathrm{Cr}$ concentration in the Cr-rich secondary phase is approximately 5.76 at $\%$ [cation ratio of $\mathrm{Cr} /(\mathrm{Al}+\mathrm{Cr})]$. On the other hand, the signal of $\mathrm{Cr}$ cannot be detected at the areas only $3 \mathrm{~nm}$ outside the Cr-rich secondary phase-alumina heterointerface. From these data, it can be considered that there is an abrupt compositional change across the interface, and the change contributes to the formation of misfit dislocations at the interface.

Theoretical configurations of misfit dislocations introduced in the Cr-rich secondary phase-alumina heterointerface can be estimated by using following geometrical relationship:

$$
D=d_{1} d_{2} /\left(d_{2}-d_{1}\right)
$$

where $D$ is an ideal interval of equivalent misfit dislocations, $d_{1}$ is the lattice constant in the alumina matrix and $d_{2}$ is the lattice constant in the Cr-rich secondary phase. For instance, ideal misfit dislocation networks introduced in such heterointerfaces can be schematically drawn by following steps. Figures 5(a) and 5(b) represent the schematic illustration of the $\{1 \overline{1} 00\}$ planes in the alumina matrix and the Cr-rich secondary phase, where the difference between their scales is exaggerated for emphasis $\left(d_{2}=1.1 \times d_{1}\right)$ in order to illustrate the misfit dislocation configurations easily. Oxygen ions are not drawn in these

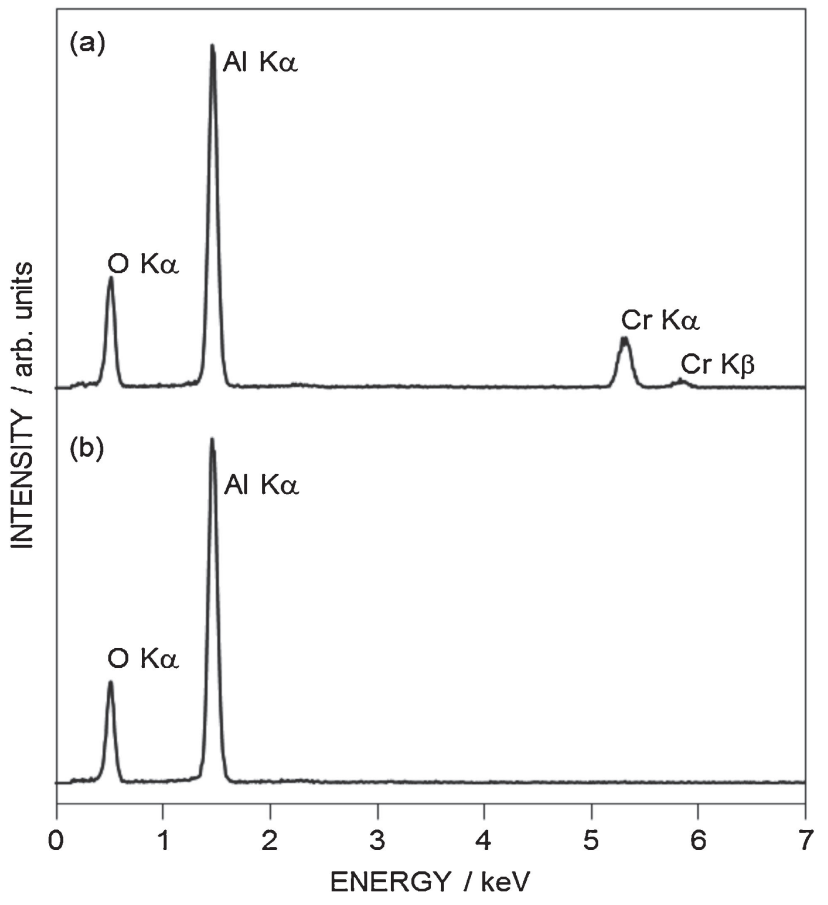

Fig. 4. (a) TEM-EDS spectrum obtained at an area in the Cr-rich secondary phase. $\mathrm{CrK}_{\alpha}$-line and $\mathrm{CrK}_{\beta}$-line can be clearly recognized. (b) TEM-EDS spectrum obtained at an area $3 \mathrm{~nm}$ outside the Cr-rich secondary phase-alumina heterointerface. Cr signals cannot be detected.

schematic illustrations. Figure 5(c) is an overlapped figure of these planes. Black circles and open circles indicate cation sites in the alumina matrix and Cr-rich secondary phase, respectively. The smallest translation vectors of alumina toward the [1120] direction is $1 / 3[11 \overline{2} 0](0.476 \mathrm{~nm})$ and toward the [0001] direction is [0001] $(1.299 \mathrm{~nm})$, and the locations of misfit perfect dislocations are represented by the orthogonal lines in the Fig. 5(c). The $\mathrm{Cr}$ concentration in the Cr-rich secondary phase are found to be about $6 \%$ by TEM-EDS analysis, and the lattice constant of which can be roughly estimated to be $0.2 \%$ larger than that of alumina. Thus, it can be calculated by Eq. (4) that misfit dislocations which have the interval of about $0.2 \mu \mathrm{m}$ toward the $[11 \overline{2} 0]$ direction and about $0.6 \mu \mathrm{m}$ toward the [0001] direction are to be introduced ideally in the Cr-rich secondary phase-alumina heterointerface.

In order to confirm the real misfit dislocation structures formed in the Cr-rich secondary phase-alumina heterointerface, TEM observation was conducted from the $\langle 1 \overline{1} 00\rangle$ plan-view direction. Figure 6 shows a typical bright-field TEM image of the heterointerface. The formation of very complex misfit dislocation network structures is seen. Extra half-planes of the dislocations should exist in the alumina matrix side of the heterointerface, because the lattice constant is smaller in the side. These network structures are not consistent with ideal structures which are shown in Fig. 5(c). Here, it can be seen that all the misfit dislocations dissociate into partial dislocations, and the dissociation width can be measured to be approximately $12-16 \mathrm{~nm}$. Stacking faults must be introduced in between these dissociated partial dislocations on the $\{1 \overline{1} 00\}$ plane of the Cr-rich secondary phase-alumina heterointerface. The remarkable difference between the ideal and the real dislocation configurations must be caused by the dissociation of the misfit dislocations, the curvature of the heterointerface and the slight distribution in $\mathrm{Cr}$ concentration. According to the previous reports, the stacking fault 

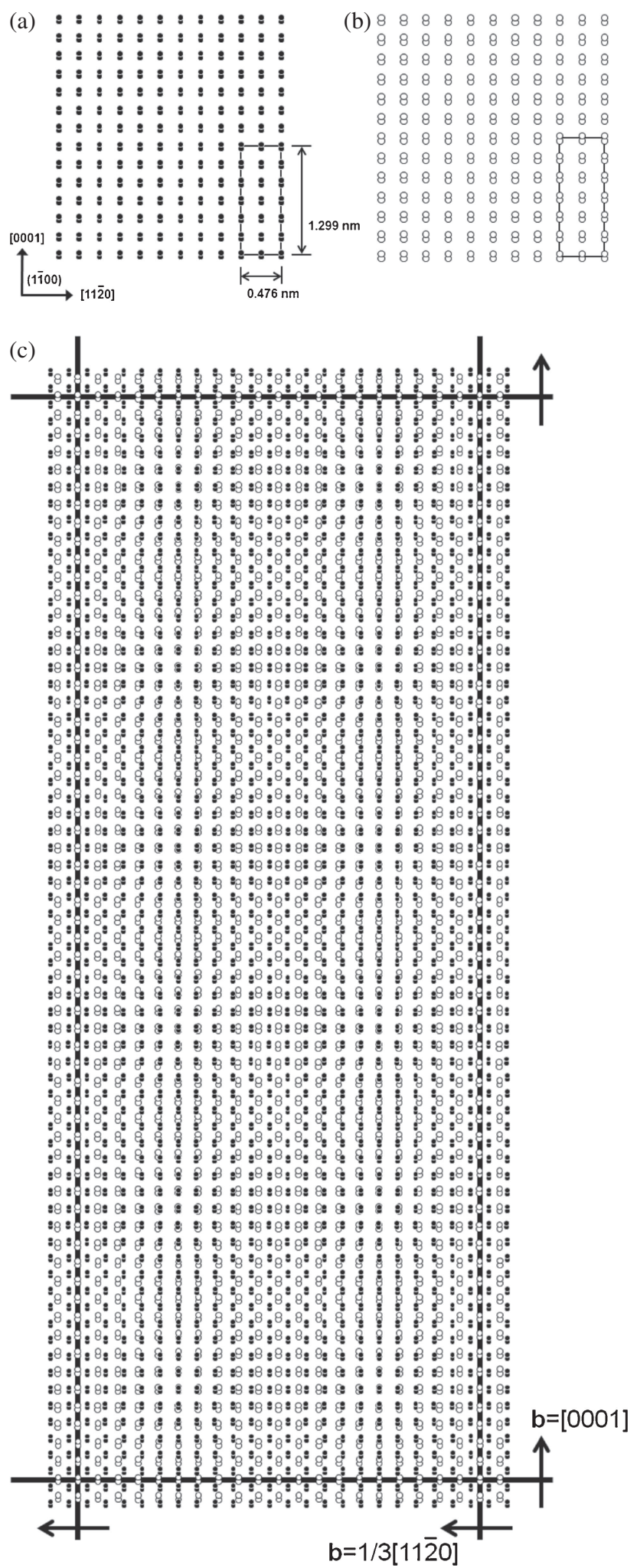

Fig. 5. The schematic illustrations of the $\{1 \overline{1} 00\}$ planes (a) in the alumina matrix and (b) in the Cr-rich secondary phase where oxygen ions are not drawn. (c) An overlapped figure of these planes. Positions of the ideal misfit perfect dislocations are represented by the orthogonal lines.

energy of pure alumina $\{1 \overline{1} 00\}$ plane is estimated to be $0.33-$ $0.46 \mathrm{~J} / \mathrm{m}^{2}$ by experiments and to be $0.41-0.46$ by first principle calculations, and the distance between the dissociated partial dislocations are reported to be normally up to $6 \mathrm{~nm}^{27)}$ Thus, it

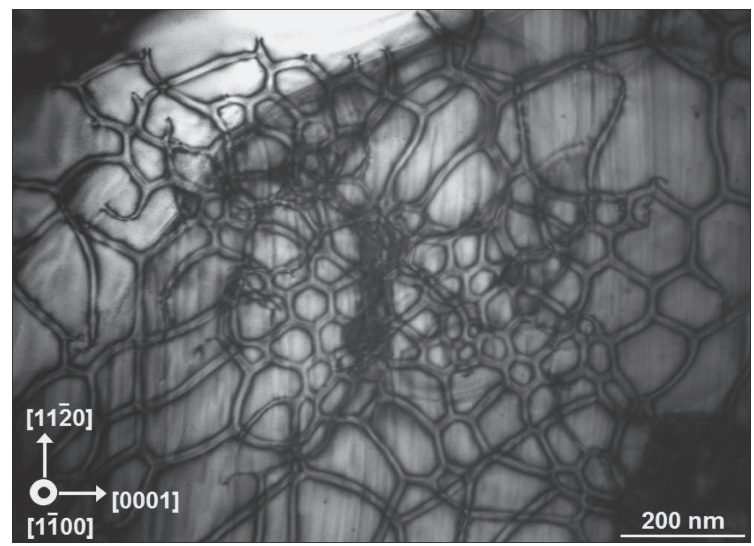

Fig. 6. Typical bright-field TEM image of the Cr-rich secondary phasealumina heterointerface observed from the $\langle 1 \overline{1} 00\rangle$ plan-view direction. The formation of complex misfit dislocation network structures was observed. It can be seen that all the misfit dislocations introduced in the heterointerface dissociate into partial dislocation pairs.

can be said that the dissociation width we observed in this study is about two times larger compared with the dislocation dissociation distances in alumina $\{1 \overline{1} 00\}$ planes reported so far. Although further detailed analysis for the dislocation structures are crucially needed, the present results suggest that the stacking fault energy of the heterointerface plane may be significantly lowered by the presence of $\mathrm{Cr}$ ions on condition that the elastic properties of the alumina and the Cr-rich secondary phase are similar.

\section{Summary}

Dislocation structures formed in the Cr-doped $\{1 \overline{1} 00\} /[0001]$ $2^{\circ}$ low-angle tilt grain boundary were investigated by TEM. A Cr-rich secondary phase with approximately $200 \mathrm{~nm}$ width was formed along the grain boundary, and an array of triply dissociated grain boundary dislocations was introduced in the middle of the Cr-rich secondary phase in order to compensate for the mistilt angle of the two bonded crystals. In addition, misfit dislocations were introduced in the Cr-rich secondary phasealumina heterointerface. TEM-EDS analysis revealed that approximately $6 \%$ of $\mathrm{Al}$ sites in the Cr-rich secondary phase were substituted by $\mathrm{Cr}$ ions, and by the abrupt $\mathrm{Cr}$ compositional change at the interface, misfit dislocations were introduced in the Cr-rich secondary phase-alumina heterointerface. The heterointerface was observed from the plan-view direction, and the formation of very complex misfit dislocation networks was observed. Misfit dislocations were found to dissociate into partial dislocations with 12 to $16 \mathrm{~nm}$ intervals, which width was rather wide compared to the stacking fault width formed in alumina $\{1 \overline{1} 00\}$ planes reported so far.

Acknowledgements A part of this work was supported by the Grant-in-Aid for Scientific Research on Priority Areas "Nano Materials Science for Atomic-scale Modification" (No. 19053001) from the Ministry of Education, Culture, Sports, Science and Technology (MEXT) of Japan. E.T. was supported as a Japan Society for the Promotion of Science (JSPS) research fellow.

\section{References}

1) T. H. Maiman, Phys. Rev., 123, 1145-1150 (1961).

2) T. H. Maiman, R. H. Hoskins, I. J. Haenens, C. K. Asawa and V. Evtuhov, Phys. Rev., 123, 1151-1157 (1961). 
3) S. Sugano and Y. Tanabe, J. Phys. Soc. Jpn., 13, 880-899 (1958).

4) S. Sugano and I. Tsujikawa, J. Phys. Soc. Jpn., 13, 899-910 (1958).

5) D. S. McClure, J. Chem. Phys., 36, 2757-2779 (1962).

6) R. A. Forman, G. J. Piermarini, J. D. Barnett and S. Block, Science, 176, 284-285 (1972).

7) G. J. Piermarini, S. Block, J. D. Barnett and R. A. Forman, J. Appl. Phys., 46, 2774-2780 (1975).

8) H. K. Mao, P. M. Bell, J. W. Shaner and D. J. Steinberg, J. Appl. Phys., 49, 3276-3283 (1978).

9) Y. M. Gupta and Z. A. Shen, Appl. Phys. Lett., 58, 583-585 (1991).

10) J. H. Eggert, K. A. Goettel and I. F. Silvera, Phys. Rev. B, 40, 5724-5732 (1989).

11) J. H. Eggert, K. A. Goettel and I. F. Silvera, Phys. Rev. B, 40, 5733-5738 (1989).

12) C. Moon, S. Kanehira, M. Nishi, K. Miura, T. Nakaya, E. Tochigi, N. Shibata, Y. Ikuhara and K. Hirao, J. Am. Ceram. Soc., 92, 3118-3121 (2009).

13) D. E. McCumber and M. D. Sturge, J. Appl. Phys., 34, 16821684 (1963).

14) D. D. Ragan, R. Gustavsen and D. Schiferl, J. Appl. Phys., 72, 5539-5544 (1992).

15) N. Yu, Q. Wen, D. R. Clarke, P. C. McIntyre, H. Kung, M. Nastasi, T. W. Simpson, I. V. Mitchell and D. Li, J. Appl. Phys.,
78, 5412-5421 (1995).

16) H. Aizawa, M. Sekiguchi, T. Katsumnata, S. Komuro and T. Morikawa, Rev. Sci. Instrum., 77, 044902 (2006).

17) C. Pflitsch, D. Viefhaus and B. Atakan, Chem. Vap. Deposition, 13, 420-426 (2007).

18) S. Wang, M. W. Shao, G. Shao, H. Wang and L. Cheng, Chem. Phys. Lett., 460, 200-204 (2008).

19) X. S. Fang, C. H. Ye, X. S. Peng, Y. H. Wang, Y. C. Wu and L. D. Zhang, J. Mater. Chem., 13, 3040-3043 (2003).

20) X. S. Fang, C. H. Ye, L. D. Zhang and T. Xie, Adv. Mater., 17, 1661-1665 (2005).

21) Y. Kezuka, E. Tochigi, N. Shibata, C. Moon, S. Kanehira, K. Hirao and Y. Ikuhara, J. Ceram. Soc. Japan, 119, 620-622 (2011).

22) T. Saito, T. Hirayama, T. Yamamoto and Y. Ikuhara, J. Am. Ceram. Soc., 88, 2277-2285 (2005).

23) E. G. Moya, F. Moya, A. Sami, D. duve, D. Treheux and C. Grattepain, Philos. Mag. A, 72, 861-870 (1995).

24) T. Nakagawa, A. Nakamura, I. Sakaguchi, N. Shibata, T. Mizoguchi, T. Yamamoto, H. Haneda, N. Ohashi and Y. Ikuhara, Acta Mater., 59, 1105-1111 (2011).

25) F. C. Frank, Philos. Mag. 7th Ser., 42, 809-819 (1951).

26) G. Cliff and G. W. Lorimer, J. Microsc., 103, 203-207 (1975).

27) E. Tochigi, N. Shibata, A. Nakamura, T. Mizoguchi, T. Yamamoto and Y. Ikuhara, Acta Mater., 58, 208-215 (2010). 